\title{
Pruning the ricket thicket
}

\section{Valentin David and Myles Wolf}

Division of Nephrology and Hypertension, Department of Medicine, Center for Translational Metabolism and Health, Institute for Public Health and Medicine, Northwestern University, Chicago, Illinois, USA.

\author{
Overexpression of FGF23 results in hypophosphatemic rickets, which is \\ characterized by renal phosphate wasting, inappropriately low circulating \\ levels of the active form of vitamin $\mathrm{D}$, and skeletal abnormalities. The \\ precise mechanisms of how excess FGF23 leads to hypophosphatemic \\ rickets are not clear. In this issue of the $J C I$, Bai and colleagues demonstrate \\ that deletion or inhibition of CYP24A1, which initiates degradation of \\ the active form of vitamin D, ameliorates skeletal abnormalities in two \\ mouse models of hypophosphatemic rickets. While this work supports an \\ important role for excess CYP24A1 activity in the pathogenesis of FGF23- \\ mediated hypophosphatemic rickets, more work will need to be done before \\ CYP24A1 inhibition can be integrated into the management of patients living \\ with these diseases.
}

\section{Precise regulation of FGF23 is} required for bone health

Just the right amount of FGF23 is required to maintain healthy bone and mineral metabolism. FGF23 deficiency results in tumoral calcinosis, because loss of this factor impedes renal phosphate excretion and enables unopposed parathyroid hormone-dependent (PTH-dependent) stimulation of 1,25-dihydroxyvitamin D $\left[1,25(\mathrm{OH})_{2} \mathrm{D}\right]$ production, which together culminate in hyperphosphatemia and metastatic calcification (1). In contrast, FGF23 overload results in hypophosphatemic rickets, which is characterized by inappropriately low levels of $1,25(\mathrm{OH})_{2} \mathrm{D}$ $(2,3)$. Yet, for all we have learned about FGF23 in the past decade, the fundamental mechanisms of how FGF23 overload leads to rickets is still unclear. Is the primary driver FGF23 excess, phosphate depletion, $1,25(\mathrm{OH})_{2} \mathrm{D}$ depletion, secondary calcium depletion, a combination of these, or other factors altogether?

\section{Targeting CYP24A1 ameliorates skeletal abnormalities}

In this issue, Bai et al. shed important new light on this controversy (4). Specifically, the authors investigated the role of CYP24A1, the cytochromal enzyme that catalyzes the first step in vitamin D degradation, in the pathogenesis of two prototypical disorders of primary FGF23 excess: X-linked hypophosphatemic rickets $(\mathrm{XLH})$, which is caused by mutations in PHEX that lead to elevated FGF23 through unknown mechanisms, and autosomal dominant hypophosphatemic rickets (ADHR), which results from activating mutations in FGF23 itself. Bai and colleagues crossed $H y p$ mice, which are the murine homolog of human XLH and FGF23 transgenic mice, which overexpress the cleavage-resistant form of FGF23 (FGF23 ${ }^{\mathrm{R} 176 \mathrm{Q}}$ ) produced in human ADHR, with Cyp24a1-null mice. As a secondary approach to evaluate the involvement of CYP24A1 in these diseases, Hyp

\section{Related Article: p. 667}

Conflict of interest: V. David reports receiving research support from Keryx Biopharmaceuticals. M. Wolf reports receiving consulting fees from or has an ownership interest in Amgen, Keryx Biopharmaceuticals, Pfizer, and Ultragenyx and is an inventor on a patent application focused on targeting FGF receptor 4 to attenuate cardiovascular disease.

Reference information: J Clin Invest. 2016;126(2):473-476. doi:10.1172/JCI85005.

and ADHR mice were also treated with a pharmacological CYP24A1 inhibitor (4).

Using this experimental approach, Bai and colleagues demonstrated that either genetic deletion or pharmacological inhibition of CYP24A1 induces near-complete healing of rickets in $H y p$ and ADHR mice. Amelioration of the animals' skeletal defects occurred in the absence of correcting severe hypophosphatemia or altering serum calcium levels. On the basis of these results, Bai et al. conclude that inactivation of CYP24A1 heals the skeleton by prolonging the local half-life of $1,25(\mathrm{OH})_{2} \mathrm{D}$ in bone, such that even low levels of circulating and locally produced $1,25(\mathrm{OH})_{2} \mathrm{D}$ have protracted effects (4). The stable serum mineral levels observed in these models are especially noteworthy, because a large amount of calcium and phosphate must have been deposited in bone to achieve such substantial remineralization. Most likely, Cyp24a1 deletion induced a major increase in gastrointestinal absorption of calcium and phosphate due to prolonged effects of $1,25(\mathrm{OH})_{2} \mathrm{D}$ in enterocytes. Although Bai and colleagues did not directly explore this mechanism by performing metabolic balance studies, their results indirectly support this hypothesis, as circulating levels of PTH and $1,25(\mathrm{OH})_{2} \mathrm{D}$ were substantially decreased, and expression of vitamin $\mathrm{D}$-dependent calcium transporters in the gut of the compound mutant mice was increased (4). Direct suppression of PTH by prolonged $1,25(\mathrm{OH})_{2} \mathrm{D}$ activity in the parathyroid glands is another possible mechanism to explain the low PTH and thus low systemic $1,25(\mathrm{OH})_{2} \mathrm{D}$ levels; however, this scenario is less likely, because primary suppression of $\mathrm{PTH}$ in the setting of massive bone calcium deposition would have resulted in severe hypocalcemia, unlike what Bai et al. report. Moreover, FGF23 levels were further increased in the compound $H y p$ Cyp24a1-null mice compared with levels 
A

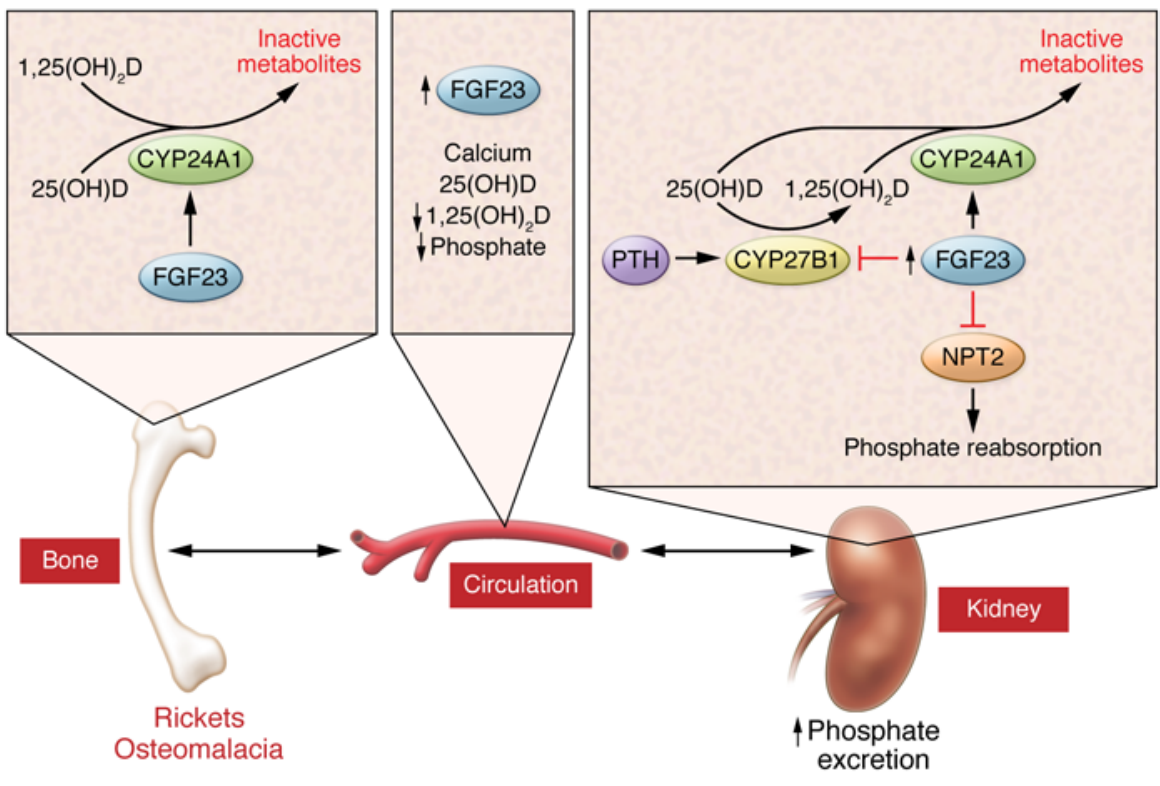

B

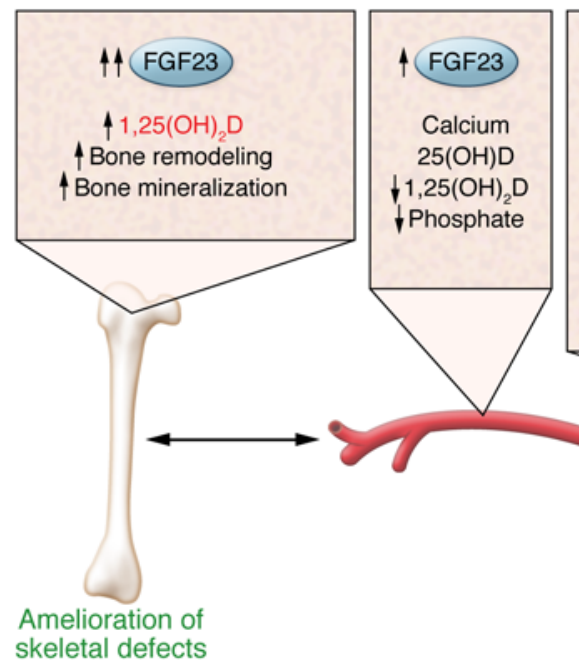

detected in the Hyp mice, perhaps reflecting a consequence of positive phosphate and calcium balance induced by the prolonged effects of $1,25(\mathrm{OH})_{2} \mathrm{D}$ locally in enterocytes and/or the further stimulation of $\mathrm{Fgf23}$ transcription by the prolonged local effects of $1,25(\mathrm{OH})_{2} \mathrm{D}$ in osteocytes.

The totality of the data generally support the hypothesis that there is a direct and beneficial effect of suppressing bone CYP24A1 on the skeletal defects in hypophosphatemic rickets. However, an important limitation of the Bai et al. study is the inability to definitively implicate bone CYP24A1 as the cause of the phenotype as opposed to secondary effects conferred by global Cyp24a1 deletion and administration of a systemically active pharmacological inhibitor of CYP24A1. Bone-specific Cyp24a1 deletion will be needed to definitively show that it is CYP24A1 in this organ that mediates the restoration of normal skeletal structure in their models. Regardless of the precise mechanism, by demonstrating that the skeletal abnormalities of hypophosphatemic rickets can be corrected without lowering elevated FGF23 or raising depressed serum phosphate levels, Bai and colleagues have provided important new evidence against these factors as direct mechanistic contributors to bone disease in XLH, ADHR, and other related conditions mediated by primary increases in FGF23 (4).
Figure 1. Vitamin $D$ functions are regulated by a balance of $1,25(\mathrm{OH})_{2} \mathrm{D}$ synthesis and degradation, both systemically and locally, in vitamin $\mathbf{D}$ target tissues. (A) In syndromes of FGF23-dependent hypophosphatemic rickets, excess FGF23 production by bone enters the circulation and reduces systemic vitamin $D$ levels through two independent mechanisms in the kidney. FGF23 induces CYP24A1 activity, which converts vitamin D storage $[25(\mathrm{OH}) \mathrm{D}]$ and active $\left[1,25(\mathrm{OH})_{2} \mathrm{D}\right]$ forms to inactive metabolites, and it inhibits CYP27B1, which is responsible for converting $25(\mathrm{OH}) \mathrm{D}$ to $1,25(\mathrm{OH})_{2} \mathrm{D}$. These effects of FGF23 are counter-regulated by the opposing effects of PTH. FGF23 also promotes renal phosphate wasting by reducing the expression of sodium-dependent phosphate cotransporters (NPT2) in the kidney and thereby reducing phosphate reabsorption. Hypophosphatemic rickets results as a consequence of these metabolic perturbations, but the primary mediator of the skeletal disease is unclear. (B) Inhibition of Cyp24a1 in murine models of FGF23dependent hypophosphatemic rickets ameliorates the skeletal defects in these animals. This is thought to result from elevated levels of $1,25(\mathrm{OH})_{2} \mathrm{D}$ locally in the bone, since systemic levels of $1,25(\mathrm{OH})_{2} \mathrm{D}$, serum phosphate, and calcium are unchanged. These results suggest a pivotal role for FGF23-mediated upregulation of CYP24A1 in the pathogenesis of hypophosphatemic rickets.

\section{Roles of circulating and local vitamin D metabolites}

It should be noted that Bai et al. did not study the possible effects of other vitamin $\mathrm{D}$ metabolites. By catalyzing hydroxylation of 25-hydroxyvitamin D [25(OH)D] to 24,25-dihydroxyvitamin D $\left[24,25(\mathrm{OH})_{2} \mathrm{D}\right]$, CYP24A1 reduces the amount of $25(\mathrm{OH})$ $\mathrm{D}$ that CYP27B1 can convert into the more potent $1,25(\mathrm{OH})_{2} \mathrm{D}(5,6)$. CYP24A1 also reduces the half-life of previously synthesized $1,25(\mathrm{OH})_{2} \mathrm{D}$ by converting $1,25(\mathrm{OH})_{2} \mathrm{D}$ into $1,24,25(\mathrm{OH})_{3} \mathrm{D}$, which is further metabolized into calcitroic acid (1-hydroxy23-carboxyvitamin D) or $1 \alpha, 25-(\mathrm{OH})_{2} \mathrm{D} 3-$ 26,23-lactone (7, 8). Consistently, previous reports have demonstrated a total absence of calcitroic acid and $1 \alpha, 25-(\mathrm{OH})_{2} \mathrm{D} 3-26,23-$ 
lactone formation in Cyp24a1-KO mice (9). Since $1 \alpha, 25-(\mathrm{OH}) 2 \mathrm{D} 3-26,23-$ lactone can act as a vitamin $\mathrm{D}$ receptor antagonist and thus inhibit osteoclastogenesis and bone resorption (10), the extent to which rescue of the bone phenotype observed by Bai and colleagues can be explained entirely by the increase in the half-life of local 1,25(OH) ${ }_{2} \mathrm{D}$ or by an additional contribution of the concomitant absence of $1 \alpha, 25-(\mathrm{OH}) 2 \mathrm{D} 3-26$, 23-lactone is not certain.

As Cyp24a1-null mice are unable to degrade $1,25(\mathrm{OH})_{2} \mathrm{D}$, it is somewhat surprising that $1,25(\mathrm{OH})_{2} \mathrm{D}$ levels are low and hypercalcemia is absent in these animals. Indeed, approximately $50 \%$ of Cyp24a1$\mathrm{KO}$ mice die in the early postnatal period due to endogenous $1,25(\mathrm{OH})_{2} \mathrm{D}$ intoxication and consequent hypercalcemia (11). For reasons that are not clear, the other $50 \%$ are capable of lowering their circulating $1,25(\mathrm{OH})_{2} \mathrm{D}$ levels to the point that hypercalcemia can be avoided and prolonged survival is possible (11). Thus, another important limitation of the study by Bai and colleagues is that only the progeny of Cyp24a1-KO mice that successfully adapted their vitamin $\mathrm{D}$ homeostasis to survive can be studied. Is it appropriate to proclaim skeletal success when only the hypercalcemia-resistant half of the progeny were analyzed? As a corollary, do the results understate the risks related to hypercalcemia in the event that all-comer human hypophosphatemic rickets patients are treated with CYP24A1 inhibitors?

In addition to highlighting the pathogenic role of excessive CYP24A1 activity in hypophosphatemic rickets, the study by Bai and colleagues emphasizes important differences between systemic and local regulation of vitamin $\mathrm{D}$ and the delicate balance between $1,25(\mathrm{OH})_{2} \mathrm{D}$ synthesis and degradation (Figure 1). The kidney is the primary source of the circulating pool of $1,25(\mathrm{OH})_{2} \mathrm{D}$. Systemically, PTH is the primary factor that increases circulating $1,25(\mathrm{OH})_{2} \mathrm{D}$ levels by stimulating renal expression of CYP27B1, and FGF23 is the main inhibitor of renal $1,25(\mathrm{OH})_{2} \mathrm{D}$ production (12). Locally, tissues can produce their own $1,25(\mathrm{OH})_{2} \mathrm{D}$ from $25(\mathrm{OH}) \mathrm{D}$, as is well described in parathyroid chief cells, keratinocytes, and macrophages (13). The observation by Bai and colleagues that systemic $1,25(\mathrm{OH})_{2} \mathrm{D}$ levels were low but the tissue effects were pronounced clearly demonstrate the dichotomy between local and systemic vitamin $\mathrm{D}$ regulation and the potential pitfalls of interpreting end-organ effects of vitamin D on the basis of circulating $1,25(\mathrm{OH})_{2} \mathrm{D}$ levels. Furthermore, regulation of $1,25(\mathrm{OH})_{2} \mathrm{D}$ synthesis has historically garnered far more attention than its degradation. The recent description of human mutations in CYP24A1 as the cause of previously idiopathic cases of hypercalcemia in newborns and adults has cast new light on the importance of the degradation pathway in health and disease that is elegantly emphasized by Bai et al. $(14,15)$.

\section{Conclusions and future directions}

The current standard of care for XLH and ADHR includes the administration of oral $1,25(\mathrm{OH})_{2} \mathrm{D}$ and phosphate supplements; however, these treatments, which address downstream consequences of the diseases rather than the root causes, are only partially effective and often poorly tolerated $(16,17)$. Novel strategies for these diseases are currently under development. For example, neutralizing antibodies against FGF23 directly target the underlying molecular mechanism of disease (18), and FGF receptor antagonists inhibit the end-organ effects of FGF23 (19). While the results of the current study by Bai and colleagues suggest that targeting CYP24A1 may be another viable therapeutic strategy, especially to correct the underlying skeletal defects, the inability to correct hypophosphatemia suggests that CYP24A1 antagonists may need to be relegated to an adjunctive role. Furthermore, the finding that circulating $1,25(\mathrm{OH})_{2} \mathrm{D}$ levels increased significantly in $H y p$ mice that were treated with the CYP24A1 inhibitor raises the question as to whether exogenous calcitriol treatment, which was not tested by Bai et al., could have achieved similar skeletal success. More in-depth research will be needed to define a new and improved standard of care for XLH, ADHR, and related conditions.

Beyond those orphan diseases of primary FGF23 excess, could there be a role for CYP24A1 inhibition in diseases of secondary FGF23 excess, such as chronic kidney disease? If elevated FGF23 accelerates $1,25(\mathrm{OH})_{2} \mathrm{D}$ degradation by stimulating CYP24A1 in extra-renal tissues, as it appears to in bone, treating kidney disease patients with nutritional vitamin D supplementation or exogenous $25(\mathrm{OH}) \mathrm{D}$ may be only of marginal benefit, because excessive local CYP24A1 activity would degrade most of the $25(\mathrm{OH}) \mathrm{D}$ and $1,25(\mathrm{OH})_{2} \mathrm{D}$ that managed to make it to target cells. Likewise, exogenous $1,25(\mathrm{OH})_{2} \mathrm{D}$ that raises systemic $1,25(\mathrm{OH})_{2} \mathrm{D}$ levels might only serve to further upregulate CYP24A1 at the tissue level, resulting in minimal changes in end-organ effects of $1,25(\mathrm{OH})_{2} \mathrm{D}$. It is into this gap that CYP24 inhibitors may ultimately prove to have a unique and potentially exciting profile to boost end-organ $1,25(\mathrm{OH})_{2} \mathrm{D}$ effects, without increasing serum phosphate or calcium levels. Stay tuned for more progress in a dynamic and rapidly evolving field.

\section{Acknowledgments}

V. David is supported by NIH grant R01DK102815, and M. Wolf is supported by NIH grants R01DK076116 and K24DK093723.

Address correspondence to: Myles Wolf, 633 N. St. Clair St., Suite 18-089, Chicago, Illinois 60611, USA. Phone: 312.503.8013; E-mail: myles.wolf@northwestern.edu.

1. Benet-Pages A, Orlik P, Strom TM, LorenzDepiereux B. An FGF23 missense mutation causes familial tumoral calcinosis with hyperphosphatemia. Hum Mol Genet. 2005;14(3):385-390.

2. ADHR-Consortium. Autosomal dominant hypophosphataemic rickets is associated with mutations in FGF23. Nat Genet. 2000;26(3):345-348.

3. Shimada T, et al. Targeted ablation of Fgf23 demonstrates an essential physiological role of FGF23 in phosphate and vitamin D metabolism. J Clin Invest. 2004;113(4):561-568.

4. Bai X, et al. CYP24 inhibition as a therapeutic target in FGF23-mediated renal phosphate wasting disorders. JClin Invest. 2016;126(2):667-680.

5. Okuda K, Usui E, Ohyama Y. Recent progress in enzymology and molecular biology of enzymes involved in vitamin D metabolism. J Lipid Res. 1995;36(8):1641-1652.

6. Ohyama Y, et al. Structural characterization of the gene encoding rat 25-hydroxyvitamin D3 24-hydroxylase. Biochemistry. 1993;32(1):76-82.

7. Ishizuka S, Norman AW. Metabolic pathways from 1 alpha,25-dihydroxyvitamin D3 to 1 alpha,25-dihydroxyvitamin D3-26,23-lactone. Stereo-retained and stereo-selective lactonization. J Biol Chem. 1987;262(15):7165-7170.

8. Reddy GS, Tserng KY. Calcitroic acid, end product of renal metabolism of 1,25-dihydroxyvitamin D3 through C-24 oxidation pathway. Biochemistry. 1989;28(4):1763-1769. 
9. Masuda S, et al. Altered pharmacokinetics of 1 $\alpha, 25$-dihydroxyvitamin D3 and 25-hydroxyvitamin D3 in the blood and tissues of the 25-hydroxyvitamin D-24-hydroxylase (Cyp24a1) null mouse. Endocrinology. 2005;146(2):825-34.

10. Ishizuka S, et al. $1 \alpha, 25$-Dihydroxyvitamin D3[1 alpha,25-(OH)2D3]-26,23-lactone inhibits 1,25-(OH)2D3-mediated fusion of mouse bone marrow mononuclear cells. Endocrinology. 1988;123(2):781-786.

11. St-Arnaud R, et al. Deficient mineralization of intramembranous bone in vitamin D-24hydroxylase-ablated mice is due to elevated 1,25-dihydroxyvitamin D and not to the absence of 24,25-dihydroxyvitamin D. Endocrinology.
2000;141(7):2658-2666.

12. Martin A, David V, Quarles LD. Regulation and function of the FGF23/klotho endocrine pathways. Physiol Rev. 2012;92(1):131-155.

13. Zehnder D, et al. Extrarenal expression of 25-hydroxyvitamin d(3)-1 $\alpha$-hydroxylase. J Clin Endocrinol Metab. 2001;86(2):888-894.

14. Schlingmann KP, et al. Mutations in CYP24A1 and idiopathic infantile hypercalcemia. N Engl JMed. 2011;365(5):410-421.

15. Tebben PJ, et al. Hypercalcemia, hypercalciuria, and elevated calcitriol concentrations with autosomal dominant transmission due to CYP24A1 mutations: effects of ketoconazole therapy. JClin Endocrinol Metab. 2012;97(3):E423-E427.

16. Costa T, et al. X-linked hypophosphatemia: effect of calcitriol on renal handling of phosphate, serum phosphate, and bone mineralization. J Clin Endocrinol Metab. 1981;52(3):463-472.

17. Carpenter TO, Imel EA, Holm IA, Jan de Beur SM, Insogna KL. A clinician's guide to X-linked hypophosphatemia. J Bone Miner Res. 2011;26(7):1381-1388.

18. Carpenter TO, et al. Randomized trial of the anti-FGF23 antibody KRN23 in $\mathrm{X}$-linked hypophosphatemia. J Clin Invest. 2014;124(4):1587-1597.

19. Wohrle S, et al. Pharmacological inhibition of fibroblast growth factor (FGF) receptor signaling ameliorates FGF23-mediated hypophosphatemic rickets. J Bone Miner Res. 2013;28(4):899-911. 